\title{
Planos bioscópicos de interés protésico y su influencia en la altura coronal
}

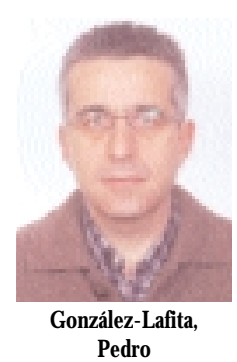

\author{
Bioscopic planes of prosthetic interest and their influence on crown height
}

\section{González- Lafita, Pedro* Álvarez-Fernández, Ma de los Ángeles** Fernández-Vázquez, J osé Pedro*** conzález- González, Ignacio**}

*Estomatólogo. Colaborador de honor del Servicio de Prótesis Estomatológica y Oclusión Facultad de Odontología. Universidad de Oviedo

**Estomatólogo, Prof. asociada del Servicio de Prótesis Estomatológica y Oclusión Facultad de Odontología. Universidad de Oviedo

***Estomatólogo, Profesor titular interino del Servicio de Prótesis Estomatológica y Oclusión Facultad de Odontología. Universidad de Oviedo
Resumen: Introducción: La correcta determinación del plano oclusal es necesaria para la elaboración de restauraciones protésicas que afecten a los sectores posteriores. El objetivo de este trabajo es verificar que parte del borde posterior del trago nos permite trazar el plano protésico más paralelo al plano oclusal, así como la influencia de dicha elección en la altura de las coronas clínicas. Material y método: Se efectuaron 40 telerradiografías laterales de cráneo a otros tantos sujetos (17 mujeres y 23 hombres). A cada participante se le habían colocado previamente tres testigos radiológicos en el trago y uno en la base del ala nasal. En cada radiografía se determinó el paralelismo del plano oclusal con cada una de las tres líneas ala de la nariz-trago trazadas (parte superior, media e inferior del trago) y la modificación de la altura de la corona clínica del primer molar superior según la línea ala de la nariz-trago tomada como referencia. A los resultados se les aplicaron métodos estadísticos. Resultados: La línea de Camper trazada por la parte inferior del trago fue la más paralela, seguida de la trazada por la parte media y de la trazada por la parte superior; entre los tres procedimientos hubo diferencias significativas. La altura clínica de la corona del primer molar superior fue 1,53 mm más corta al tomar como referencia el punto medio del trago y 2,93 más corta al tomar como referencia el punto superior; estas diferencias también fueron significativas. Conclusión: La línea de Camper que pasa por la parte inferior del trago es la más paralela con el plano oclusal y determina una corona clínica en el primer molar superior significativamente más larga que los trazados por la parte superior y media del trago.

Palabras clave: Plano de Camper, Plano oclusal, Plano protésico, Altura coronal.

Abstract: Introduction: The exact determination of the occlusal plane is necessary to construct proper prosthetic restorations involving posterior areas. The goal of our study is to determine which part of the posterior edge of the tragus enables the tracing of the prosthetic plane which is most parallel to the occlusal plane as well as the influence that this selection has on the height of the clinical crowns. Material and method: 40 lateral tele-radiographs were taken on 40 subjects (17 women, 23 men). Previously, on each participant three radiological markers had been placed on the tragus and one on the base of the wing of the nose. The parallelism between the lines traced from each tragus marker and the nose base marker plane (upper part, mid part and lower part of the tragus), and changes on the height of the clinical crown of the first upper molar were then determined. Results: The most parallel plane to the occlusal plane was the Camper plane traced through the lower part of the tragus, followed by that traced through the mid part and lastly by the one traced throughout the upper part of the tragus. Differences among procedures were statistically significant. The clinical crown height of the first upper molar was $1.53 \mathrm{~mm}$ shorter when based on the mid part of the tragus as a reference and $2.93 \mathrm{~mm}$ shorter when taking as a reference the upper part of the tragus. These differences were also signifficant. Conclusions: Camper plane traced through the lower part of the tragus is the most parallel to the occlusal plane and it creates a longer clinical crown of the first upper molar than those obtained by using the camper planes traced through the mid and upper part of the tragus, in a statistically signifficant way.

Key words: Camper plane, Occlusal plane, Prosthetic plane, Crown height

\section{Correspondencia}

Pedro González Lafita

San Bernardo 20, 1ำA

33201 Gijón (Asturias)

E-mail: pglafita@yahoo.es
BIBUD [1138-123X (2003)8:5; agosto-septiembre 469-592]

González-Lafita P, Álvarez-Fernández MA, Fernández-Vázquez JP, GonzálezGonzález I. Planos bioscópicos de interés protésico y su influencia en la altura coronal. RCOE 2003;8(5):501-507. 


\section{Introducción}

De acuerdo con el glosario de términos prostodóncicos ${ }^{1 * *}$, el plano oclusal se define como «el plano establecido por el borde incisal y la superficie oclusal de los dientes. No es realmente un plano sino que representa la media de la curvatura de las superficies».

La correcta determinación del plano oclusal en cualquier tipo de restauración -prótesis completa, fija o implantosoportada- que restituya los grupos posteriores es un importante factor para lograr el éxito desde el punto de vista estético y funcional.

Desde el punto de vista funcional, la tabla oclusal debe de estar situada de forma que la lengua y el músculo buccinador sean capaces de colocar en posición correcta el bolo oclusal durante la masticación. Si la tabla oclusal está demasiado alta se producirá un acumulo de alimentos en el fondo del surco vestibular y si está demasiado baja el paciente se morderá la mucosa yugal o la lengua ${ }^{2 *}$. Por este motivo, Merkeley ${ }^{3}$ y Lunqdist y Luther ${ }^{4}$ recomiendan colocar el plano oclusal alineado con el músculo buccinador y Yasaki ${ }^{5}$ sugiere la conveniencia de que el plano oclusal esté a la altura de la lengua. Se considera que la inclinación anteroposterior del plano protésico debe de ser igual que la inclinación del plano oclusal del sujeto dentado.

Estéticamente, el plano formado por el borde de los incisivos superiores debe de seguir la curva del labio inferior ${ }^{2 * *}$.

Por otra parte, la adecuada determinación del plano oclusal, puede influir en la supervivencia a largo plazo de la prótesis y del terreno de soporte ya sean dientes naturales, mucosa o implantes. Pequeñas discrepancias con el plano protésico (proyección bioscópica del plano de Camper es decir la línea ala de la nariz-trago) que habitualmente se utiliza como referencia para determinar la inclinación y la orientación del plano oclusal, ocasionan variaciones importantes en la altura coronal de los dientes posteriores a favor de la arcada maxilar o mandibular. El empleo de la línea ala de la nariz-trago está sujeto a controversias debido, en parte, a la falta de acuerdo sobre el punto de referencia exacto para trazar esta línea. Así por ejemplo Spratley describe esta línea desde el ala de la nariz hasta el centro del trago; para otros autores $^{7 * 8}$ se debe de tomar como referencia la parte superior del borde posterior del trago e incluso hay autores que no precisan que parte del borde posterior del trago se debe emplear para trazar la línea ala-trago.

En este sentido y ante la falta de acuerdo para determinar que parte del trago es la referencia más adecuada para obviar el disparalelismo con el plano oclusal, nuestro objetivo es aclarar esta cuestión planteando la siguiente hipótesis: el plano ala de la nariz-trago que pasa por el tercio superior del trago es el que menor discrepancia tiene con el plano oclusal. Asimismo pretendemos con nuestro trabajo averiguar si las diferentes referencias empleadas para determinar el plano protésico, parte superior media o inferior del trago, producen diferencias significativas en la altura clínica de las coronas de los dientes posteriores, dada la repercusión que este aspecto puede tener tanto en la retención de las prótesis completas como en la carga transmitida a los pilares de prótesis fija, especialmente en el caso de dientes periodontales.

El conocimiento de esta posible influencia de la inclinación del plano oclusal en el tamaño de las coronas clínicas también es de interés en el caso de prótesis implantosoportada habida cuenta que en los sectores posteriores las características anatómicas del paciente nos pueden obligar a colocar implantes de poca longitud en los que es aconsejable elaborar prótesis con las coronas clínicas lo más cortas posible para mejorar la proporción coronaimplante y disminuir las cargas sobre las fijaciones intraóseas*.

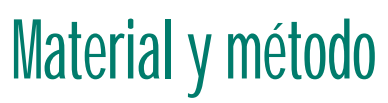

En una muestra de 40 sujetos, 17 mujeres y 23 hombres, con una edad media de 25,6 años totalmente dentados y sin patología oclusal, muscular ni articular, se efectuó, tras el preceptivo consentimiento informado, una telerradiografía lateral de cráneo (fig. 1) después de colocar en cada uno de ellos tres testigos radiológicos en el trago (parte superior media e inferior) (fig. 2) y uno en el ala de la nariz (fig. 3).

Sobre las telerradiografías trazamos (fig. 4) el plano oclusal, definido como el que pasa por la cúspide mesiovestibular de los primeros molares superiores y el borde incisal del incisivo central superior, y la línea ala de la nariz-trago, desde la parte superior inferior y media del trago al ala de la nariz.

Para evaluar el paralelismo de la línea ala de la nariz-trago con el plano oclusal, medimos el ángulo formado entre cada una de las líneas ala de la nariz-trago trazadas y la perpendicular al plano oclusal, de forma que $90^{\circ}$

RCOE, 2003, Vol 8, №5, 501-507 


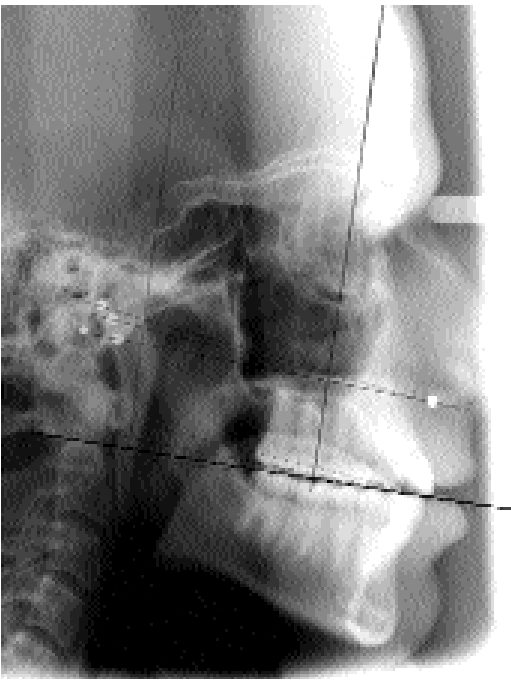

Figura 1. Telerradiografía lateral de cráneo.

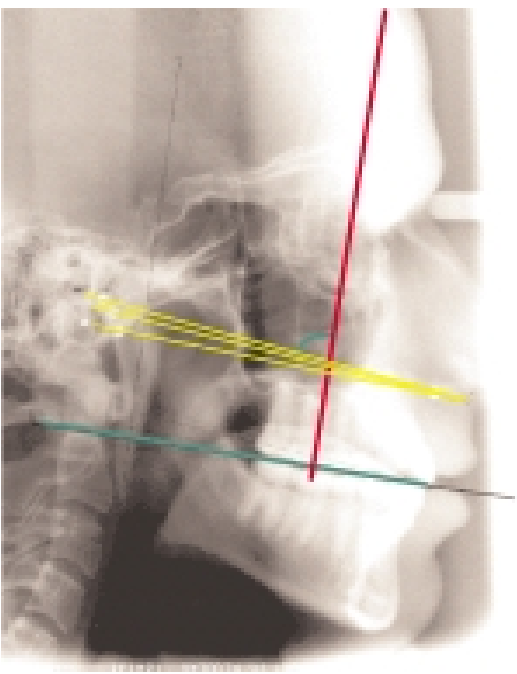

Figura 4. Procedimiento de medida del paralelismo entre el plano oclusal y la línea ala de la nariz trago.

representaba el paralelismo entre la línea ala de la nariz-trago y el plano oclusal (fig. 4).

Una vez hallada la línea más paralela con el plano oclusal, se midió la distancia entre las tres líneas ala de la nariz-trago a la altura de la cúspide mesiovestibular del primer molar

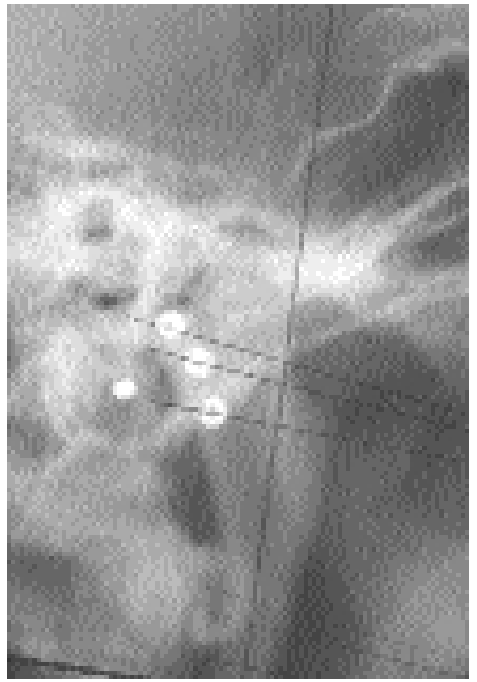

Figura 2. Detalle de la radiografía con los testigos colocados en el trago.

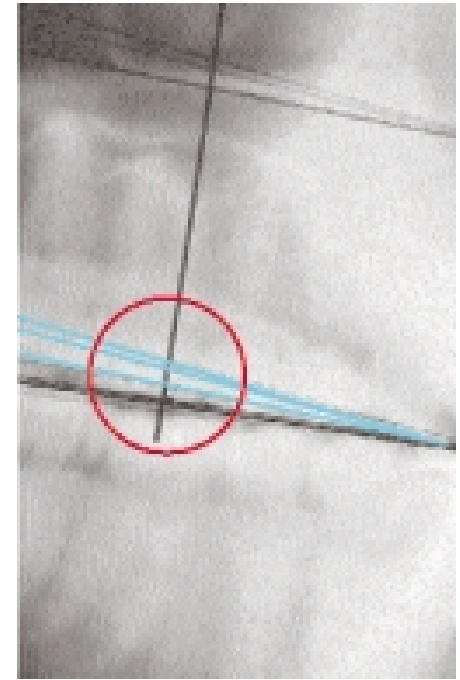

Figura 5. Variación de la altura de la corona clínica del primer molar superior.

superior, para evaluar como se modifica la altura de las coronas clínicas según el punto del trago escogido para trazar dicha línea (fig. 5) tomando como valor 0 el correspondiente a la línea ala de la nariz trago más paralela al plano oclusal.

A los datos obtenidos se les aplica-

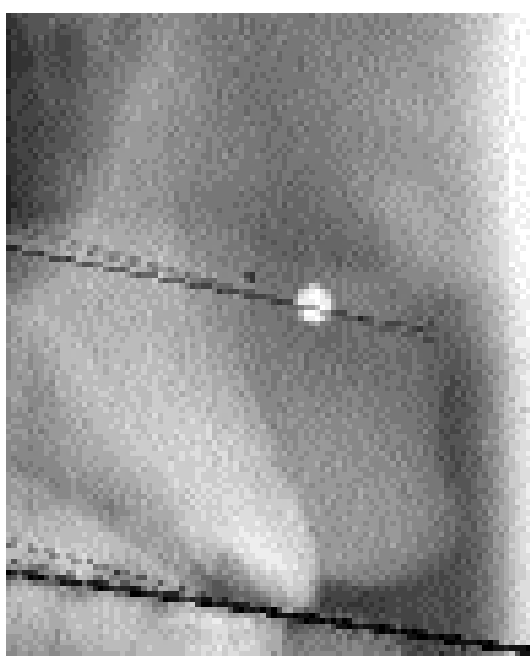

Figura 2. Detalle de la radiografia con los testigos colocados en el ala de la nariz.

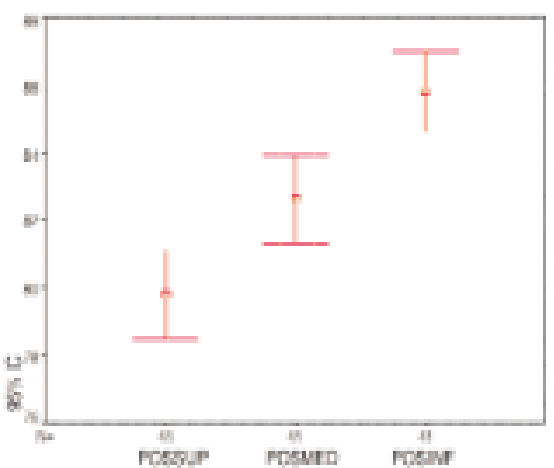

Figura 6. Medida y desviación típica de las mediciones del paralelismo entre el plano oclusal y las tres lineas de Camper trazadas.

ron pruebas estadísticas uni y multivariantes. Para contrastar el comportamiento de los puntos de referencia contrastamos la normalidad de las muestras. Cuando se verificó la hipótesis de normalidad utilizamos análisis de la varianza para muestras relacionadas. Cuando se rechazó la hipótesis 
de normalidad empleamos el contraste no paramétrico de Wilcoxon para muestras relacionadas. Se utilizó un nivel de significación del 0,001.

\section{Resultados}

Los resultados de las mediciones efectuadas están recogidos en la tabla 1. Las medidas de tendencia central y dispersión en la tabla 2

\section{Comparación de los datos}

En primer lugar se plantea un contraste para determinar si estas variables, inclinación de la línea de Camper respecto al plano oclusal y variación de la altura de la corona clínica, siguen una distribución normal (tabla 3).

\section{a. Plano oclusal}

Como se puede ver en la significación, no se rechaza la hipótesis de normalidad para un nivel de significación de 0,05, así que haremos contrastes de hipótesis suponiendo normalidad.

Para comparar los tres métodos se puede utilizar un análisis de la varianza con medidas repetidas (en cada paciente se emplean los tres métodos) cuyos resultados están recogidos en la tabla 4.

Es decir con cualquiera de los métodos de contraste que se emplee, por ejemplo la Lambda de Wilks (que es el más conocido), se rechaza la hipótesis de que los tres procedimientos sean iguales (tabla 4).

Ahora comprobamos que grupos son diferentes entre si (tabla 5).

Los tres grupos son diferentes entre si. Por lo tanto el ángulo más parecido a 90 grados es el del inferior $(90,56)$, después el medio $(87,51)$ y

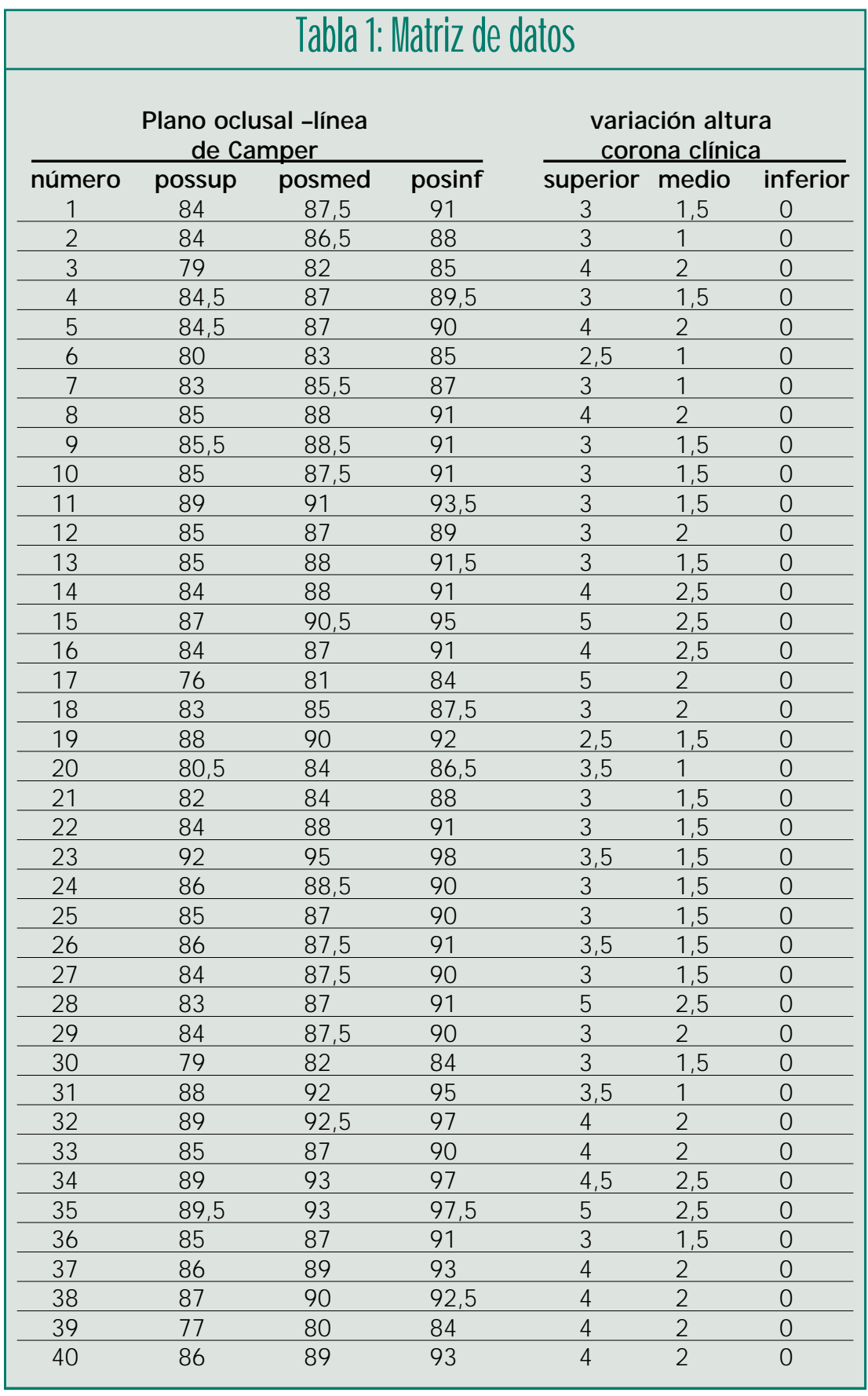

por último el superior $(84,56)$ (fig. 6). Estos resultados son estadísticamente significativos de modo que la línea de
Camper trazada por la parte inferior del trago es la más paralela al plano oclusal. 


\begin{tabular}{|c|c|c|c|c|c|}
\hline & $\mathbf{N}$ & Mínimo & Máximo & Media & Desv. típica \\
\hline possup & 40 & 76 & 92 & 84,562 & 3,3228 \\
\hline posmed & 40 & 80 & 95 & 87,512 & 3,8226 \\
\hline posinf & 40 & 84 & 98 & 90,562 & 3,5916 \\
\hline coronosup & 40 & 2,5 & 5 & 2,938 & 0,7267 \\
\hline coronomed & 40 & 1 & 2,5 & 1,538 & 0,4721 \\
\hline \multicolumn{6}{|c|}{$\begin{array}{l}\text { Possup: medida del ángulo formado entre la línea ala de la nariz-trago que pasa por } \\
\text { la parte superior de este y la perpendicular al plano oclusal. } \\
\text { Posmed: medida del ángulo formado entre la línea ala de la nariz-trago que pasa por } \\
\text { la parte media de este y la perpendicular al plano oclusal. } \\
\text { Posinf: medida del ángulo formado entre la línea ala de la nariz-trago que pasa por la } \\
\text { parte inferior de este y la perpendicular al plano oclusal. } \\
\text { Coronosup: variación en la altura de la corona del primer molar superior al trazar la } \\
\text { línea de Camper por la parte superior del trago. } \\
\text { Coronomed variación en la altura de la corona del primer molar superior al trazar la } \\
\text { línea de Camper por la parte media del trago. }\end{array}$} \\
\hline
\end{tabular}

\begin{tabular}{|lccccc|}
\hline \multicolumn{7}{|c|}{ Tabla 4: Contrastes multivariados } \\
\hline & Valor & $\mathbf{F}$ & $\begin{array}{c}\text { Gl de la } \\
\text { hipotesis }\end{array}$ & $\begin{array}{c}\text { Gl del } \\
\text { error }\end{array}$ & Significación \\
\hline Traza de Pillai &, 958 & 435,28 & 2,000 & 38,00 &, 000 \\
\hline Lambda de Wilks &, 042 & 435,28 & 2,000 & 38,00 &, 000 \\
\hline Traza de Hotelling & 22,91 & 435,28 & 2,000 & 38,00 &, 000 \\
\hline Raíz mayor de Wilics & 22,91 & 435,28 & 2,000 & 38,00 &, 000 \\
\hline
\end{tabular}

\section{b. Variación de la altura de la corona clínica}

Tomamos como referencia el plano de Camper que pasa por la parte inferior del trago por ser el más paralelo al plano oclusal y consideramos la altura de la corona oclusal en ese punto igual a cero. A partir de esa referencia medimos las variaciones en la altura de la corona clínica del primer molar superior al trazar el plano oclusal por la parte media y la parte superior del borde posterior del trago.

Como se trabaja con datos apareados, hacemos la prueba de ShapiroWilks para la variable diferencia entre corona superior y corona media (V1), rechazándose la hipótesis de normalidad (tabla 3), así que se emplearán contrastes no paramétricos como el Test de Rangos de Wilcoxon para muestras relacionadas, cuyos resultados se muestran en la tabla 6 y que nos da como resultado una $p<0,001$ con lo cual se rechaza la hipótesis de que las alturas de las coronas clínicas sean iguales. La descripción de cada grupo aparece en la tabla 2.

Por tanto, la altura de la corona clínica del primer molar superior cuando trazamos el plano de Camper por la parte media del trago es $1,54 \mathrm{~mm}$

\begin{tabular}{|lcrr|}
\hline $\begin{array}{r}\text { Tabla 3: Prueba de conformidad } \\
\text { a la distribución normal }\end{array}$ \\
\hline \multicolumn{4}{|c|}{ Shapiro-wilks } \\
Estadítico & gl & Sig \\
\hline Possup &, 952 & 40 &, 091 \\
\hline Posmed &, 961 & 40 &, 175 \\
\hline Posinf &, 950 & 40 &, 076 \\
\hline$V_{1}$ &, 863 & 40 &, 000 \\
\hline
\end{tabular}

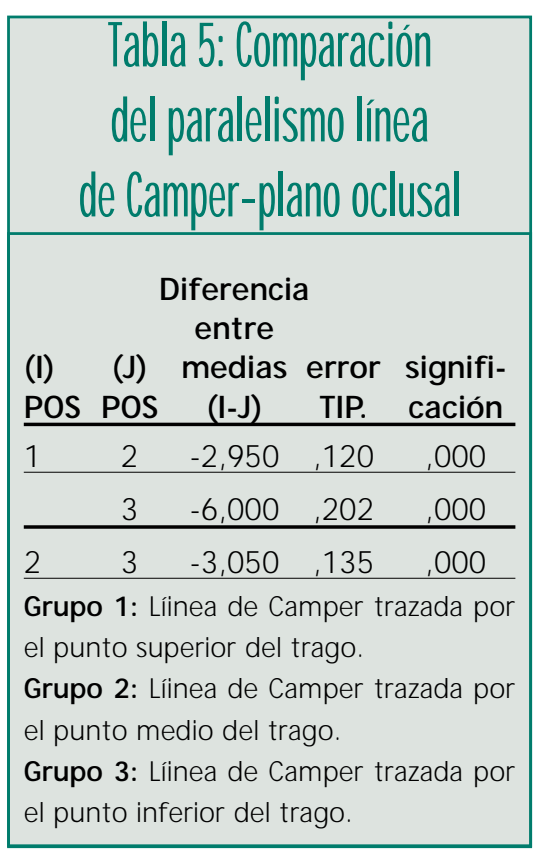

\begin{tabular}{|l}
\hline $\begin{array}{c}\text { Tabla 6: Test de Rangos de } \\
\text { Wilcoxon para muestras } \\
\text { relacionadas }\end{array}$ \\
\hline$\frac{\text { comosup-coronomed }}{\text { Sig asintót. ,000 }}$
\end{tabular}

menor que cuando la trazamos por la parte inferior; si nos referimos a la línea de Camper trazada por la parte superior, entonces la corona del pri- 
mer molar superior será 2,94 mm más corta. Estas diferencias son estadísticamente significativas (tabla 6).

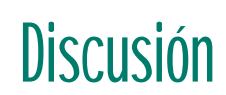

La importancia del plano oclusal ya fue reseñada por Simon en el año $1925^{10^{* *}}$. En el sresumen de términos prostodóncicos» ${ }^{1 * *}$ se define como el plano medio establecido por las caras oclusales y los bordes incisales de los dientes. La determinación de este plano, que en realidad no existe como tal puesto que las cúspides no se colocan sobre un plano, es necesaria para la elaboración de las restauraciones protésicas que reponen los dientes posteriores. Con este fin se considera que el plano oclusal es paralelo al plano de Camper, definido como el formado por el acanthion y el centro de ambos orificios óseos del CAE. Dado que esta definición del plano de Camper emplea relieves óseos, es necesario recurrir a la proyección bioscópica de los mismos para poder hallarlo en la práctica clínica. En este sentido, si bien la proyección cutánea de la espina nasal anterior ofrece pocas dificultades, no hay acuerdo entre los diferentes autores sobre que parte del trago debemos emplear para hallar la línea de Camper o línea ala-tragus, que une el ala de la nariz con algún punto del trago en general el borde ${ }^{1 * *}$ y que nos servirá para determinar la inclinación del plano protético.

Las referencias bioscópicas para su determinación son variables según los autores consultados. Para Spratley ${ }^{6}$ la línea de Camper se debe trazar desde el ala de la nariz hasta el centro del trago, mientras que otros autores ${ }^{7 *, 8}$ consideran que pasa por la parte superior del borde posterior del trago e incluso hay autores que no precisan que parte del borde posterior del trago se debe emplear para trazar la línea ala-trago.

También se ha descrito que la inclinación del plano oclusal en sujetos dentados en relación con el plano de Camper tiene una gran variación interindividual con un rango de hasta más de $20^{\circ}$ y varía de acuerdo con el tipo facial ${ }^{11^{*}}$.

D'Souza y Bhargava ${ }^{7 *}$ describen que la línea de Camper trazada por la parte superior del trago en pacientes desdentados forma un ángulo con el plano maxilar mayor que el encontrado entre el plano oclusal de pacientes dentados y el plano maxilar datos que traducen, en consonancia con lo hallado por nosotros, una excesiva inclinación de la línea de Camper con respecto al plano oclusal cuando aquella se traza por la parte superior del trago y concluyen que el empleo de la línea de Camper para determinar el plano oclusal es cuestionable.

Al contrario de lo hallado en nuestro estudio, Xie y colaboradores ${ }^{12}$ relatan un mayor paralelismo de la línea de Camper trazada por la parte media del trago y Sinobad y Postic ${ }^{13}$ recomiendan trazar la línea de Camper por la parte superior del trago en pacientes con Clase II esquelética, por la par- te media del trago en pacientes con Clase I y por la parte inferior del trago en pacientes con Clase III.

Aunque la influencia de la inclinación del plano oclusal en la altura de la corona clínica de nuestras restauraciones es conocida9*, no hemos encontrado en la literatura trabajos que cuantifiquen dicha variación. Nuestros resultados parecen indicar que podemos emplear la inclinación del plano oclusal para modificar la altura coronal de forma que disminuya la carga soportada por las raíces o implantes de peor pronóstico de modo que, si deseamos disminuir la altura de la corona del primer molar superior trazaremos la línea de Camper por la parte superior del borde posterior del trago y si, por el contrario, deseamos que las coronas clínicas de los molares inferiores sean más cortas tomaremos como referencia para trazar el plano oclusal la línea de Camper que pasa por la parte más inferior del trago.

\section{Conclusiones}

1. La línea de Camper trazada por la parte inferior del borde posterior del trago es más paralela al plano oclusal que cuando se traza por la parte media o superior del borde posterior del trago.

2. Existen diferencias significativas en la altura coronal del primer molar superior según que parte del borde posterior del trago se utilice como referencia para trazar la línea de Camper. 


\section{Bibliografía recomendada}

Para profundizar en la lectura de este tema, el/los autor/es considera/an interesantes los artículos que aparecen señalados del siguiente modo: * de interés ${ }^{* *}$ de especial interés.

$1 * *$. Van Blarcow CW. The glossary of prosthodontics termes. $7^{\text {a }}$ Ed. J Prosthet Dent 1998 ; 81:40-110.

Imprescindible y exhaustiva recopilación de los términos empleados en todo tipo de textos o comunicaciones referentes a la prótesis dental.

$2 * *$. Monteith BD. A cephalometric method to determine the angulation of the oclusal plane in edentulous patients. $\mathrm{J}$ Prosthet Dent 1985; 54:81-7.

En este artículo se hace mención de los criterios a seguir para un correcto diseño, desde el punto de vista estético y funcional, del plano oclusal así como se propone un procedimiento cefalométrico para determinar su inclinación, en el sentido anteroposterior, en pacientes desdentado.

3. Merkeley HJ. The labial and buccal accessory muscles of mastication. J Prosthet Dent 1954; 4:327-34.

4. Lundquist DO, Luther WW. Occlusal plane determination. J Prosthet Dent 1970; 23:489-98.

5. Yasaki M. The height of the oclusal rim and the interocclusal distance. $\mathrm{J}$ Prosthet Dent 1961; 11:26-31.

6. Spratley MH. A simplified technique for determining the oclusal plane in full denture construction. J Oral Rehabil 1980;7:31.

7*. D'Souza NL, Bhargava K. A cephalometric study comparing the oclusal plane in dentulous and edentulous subjects in relation to the maxilomandibular space. J Prosthet Dent 1996;75:177-82.

Los autores relacionan en este trabajo distintas variables cefalométricas con la inclinación del plano oclusal en sujetos dentados y desdentados.

8. Ismail YH, Bowman JF. Position of the occlusal plane in natural and artificial teeth. $\mathrm{J}$ Prosthet Dent 1961;20:407-11.

9*. Misch CE. Dentadura maxilar opuesta a una sobredentadura de implantes o a una prótesis fija. En: Misch $\mathrm{CE}$ ed. Implantología contemporánea. Madrid: Mosby/Doyma 1995:679-96.

Interesante y completo texto referido a distintos aspectos diagnósticos y terapéuticos con dife- rentes tipos de prótesis implantosoportadas. 10 **. Martínez Ross E. Movimientos mandibulares. En: Martínez Ross E, ed. Oclusión orgánica México: Salvat Mejicana de ediciones 1985: 79-190.

Manual de aspectos anatómicos y funcionales de la oclusión.

11*. Ogawa T, Koyano K, Suetsugu T. The relationship between inclination of the oclusal plane and jaw closing path. J Prosthet Dent 1996;76:576-80.

Estudio realizado en sujetos dentados que revela que pese a la variación en la inclinación del plano oclusal, la trayectoria de cierre mandibular tiende a mantenerse perpendicular.

12. Xie J, Zhao Y, Chao Y, Luo W. A cephalometric study determining the orientation of oclusal plane (Abstracts). Hua XI Ke Da Xue Bao 1993;24:422-5.

13. Sinobad D, Postic SD. Roentgencraniometric indicators of the position of the oclusal plane in natural and artificial dentitions Eur. J Prosthodont Restor Dent 1996;4:169-74. 